\title{
ANÁLISE DE UM EXPERIMENTO PARA COMBATE DE VIRA-CABEÇA DO TOMATEIRO(')
}

C. G. Fraga JR., engenheiro agrônomo, Seção de Técnica Experimental e Cálculo e A. S. Costa, engenheiro agrônomo, Seç̧ão de Genética, Instituto Agronômico de Campinas

\section{1 - INTRODUÇÃO}

Em experiências realizadas anteriormente (2) foi estudada a eficiência de vários inseticidas no contrôle de vira-cabeça do tomateiro, pela destruição do vetor. Nestes ensaios compararam-se concentrações progressivas de cada inseticida, procurando-se atingir a máxima suportada pela planta, sem demasiado dano. Desta maneira poder-se-ia determinar a concentração mais adequada a ser empregada, pois esta depende de sua eficiência na destruição do vetor e dos efeitos prejudiciais que o inseticida possa exercer sôbre a planta.

Com base nos resultados obtidos, nova série de ensaios foi planejada, para estudar o efeito comparativo dos diferentes inseticidas nas concentrações mais indicadas. Neste trabalho daremos os resultados obtidos no primeiro ensaio desta segunda série de experiências e sua análise estatística detalhada.

\section{2 - MATERIAL E METODO}

Os inseticidas comparados e doses respectivas acham-se incluídos na relação seguinte :

\begin{tabular}{|c|c|c|}
\hline TRATA & MENTO & $\begin{array}{l}\text { Concentrasão } \\
\text { produto comes }\end{array}$ \\
\hline $0 \ldots$ & Testemunha & prowoso \\
\hline 13 & $\begin{array}{l}\text { Rhodiatox } \\
\text { Rhodiatox } \\
\text { Rhodiatox }\end{array}$ & $\begin{array}{l}1,00 \% \\
0,50 \\
0,25 \%\end{array}$ \\
\hline $\begin{array}{l}4 \\
5 \\
5\end{array}$ & $\begin{array}{l}\text { Hexason } 2540 \mathrm{M} \\
\text { Hexason } 2540 \mathrm{M}\end{array}$ & $\begin{array}{l}0,50 \% \\
0,25 \%\end{array}$ \\
\hline 6. & $\begin{array}{l}\text { Toxafeno } \\
\text { Toxafeno }\end{array}$ & $\begin{array}{l}\mathbf{0 , 5 0} \% \\
\mathbf{0 , 2 5} \%\end{array}$ \\
\hline $\begin{array}{l}8 \\
9\end{array}$ & $\begin{array}{l}\text { Tártaro emético } \\
\text { Tártaro emético }\end{array}$ & $\begin{array}{l}\mathbf{0 , 5 0} \% \\
0,25 \%\end{array}$ \\
\hline $10 \ldots \ldots$ & $\begin{array}{l}\text { Fosfern } \\
\text { Fosfern } \\
\text { Fosfern }\end{array}$ & $\begin{array}{r}0,50 \% \\
0,25 \% \\
0,125 \%\end{array}$ \\
\hline
\end{tabular}

O princípio ativo em Rhodiatox e Fosfern é o tiofosfato de dietil paranitrofenila. Em Rhodiatox, sua concentração é de 5\%, ao passo que é de $20 \%$ em Fosfern. Devido à diferença em concentração, o tratamento 10 corres-

(1) Trabalho a ser apresentado no 5.० Seminário de Estatística, a se realizar em C.uritiba, em novembro de 19.50. 
ponde a um tratamento com 2,0\% de Rhodiatox, e os tratamentos $11 \mathrm{e}$ 12 correspondem aos tratamentos 1 e 2 (1,0\% e 0,5\% de Rhodiatox), respectivamente. Estes inseticidas foram pulverizados, tendo-se adicionado açúcar ao tártaro emético, na proporção de um por cento.

Quanto ao número de aplicações, seguiu-se a mesma orientação dos ensaios anteriores, procurando-se fazê-las com bastante frequência, a fim de que a falta de ação de determinado tratamento não pudesse ser atribuída à deficiência no número de pulverizaçzes. Foi feita uma pulverização na ocasião do transplante e as outras em intervalos de 3 a 6 dias, até 35 dias após a transplantação.

Os 13 tratamentos foram comparados em um delineamento em blocos incompletos balanceados, usando-se blocos de 4 tratamentos e 4 repetições. Cada um dos canteiros utilizados compreendia um total de 30 plantas dispostas em 3 fileiras de 10 plantas.

\section{3 - ANALISE DOS RESULTADOS EXPERIMENTAIS}

Uma experiência em blocos incompletos balanceados consiste no arranjo de $\mathbf{v}$ tratamentos em $\mathbf{b}$ blocos de $\mathbf{k}$ canteiros cada um dêles, onde $\mathbf{k}<\mathbf{v}$. Eiste delineamento obedece às mesmas condiçōes que regem os blocos ao acaso, menos àquela que requer que todos os tratamentos ocorram em todos os blocos. Esta condição é substitúda pela seguinte : Um tratamento $v_{i}$ ocorre em um mesmo bloco que o tratamento $\mathrm{v}_{\mathrm{j}}$ um número constante de vêzes. $\mathrm{Na}$ experiência que estamos considerando, os valores caraterísticos são os seguintes :

$$
\mathrm{b}=\mathrm{v}=13 ; \mathrm{r}=\mathrm{k}=4 ; \lambda=1 .
$$

As abreviações $\mathbf{r}$ e $\mathbf{k}$ representam repetições e número de tratamentos em um bloco, respectivamente, e $\lambda$ indica o número de vêzes em que dois tratamentos ocorrem em um mesmo bloco. Análises de experiências dêste tipo podem ser encontradas em Fisher e Yates (3) e Cochran e Cox (1).

\section{1 - ANALISE DO NUMERO DE PLANTAS SADIAS}

No quadro 1 representamos a distribuição dos tratamentos dentro dos blocos e o número de plantas sadias correspondente a cada tratamento.

Quadro 1.- Delineamento da experiência e número de plantas não afetadas pelo viracabeça, por canteiro de 30 plantas

\begin{tabular}{rl|r|r|r|r|r|r}
\hline \multicolumn{2}{c|}{ Número dos blocos } & \multicolumn{5}{|c}{ Dísposição dos canteiros e respectivos números de plantas sadias } & $\begin{array}{r}\text { Número } \\
\text { total de } \\
\text { plantas }\end{array}$ \\
\hline 1 & &
\end{tabular}


Essses resultados devem ser considerados, para a análise estatística, como sendo proporções entre o número de plantas sadias e o número total de plantas. Por conseguinte, não serão analisados sob essa forma, mas após a transformação, de acôrdo com a seguinte fórmula (4) :

$$
\mathrm{p}=\operatorname{sen}^{2} \varphi \text {. }
$$

O quadro 2 contém os dados transformados.

QUADRo 2.-Ângulo correspondente ao número de plantas sadias

\begin{tabular}{|c|c|c|c|c|c|c|c|c|c|c|c|c|c|c|}
\hline \multirow[b]{2}{*}{ Bloco } & \multicolumn{13}{|c|}{$T$ R A T A M E N T O S } & \multirow[b]{2}{*}{ Total } \\
\hline & 0 & l & 2 & 3 & 4 & 5 & 6 & 7 & 8 & 9 & 10 & 11 & 12 & \\
\hline $\begin{array}{l}1 \\
2 \\
2 \\
3 \\
4 \\
5\end{array} \ldots$ & $\begin{array}{l}52,7 \\
88,9 \\
-\ldots\end{array}$ & $\begin{array}{c}79,5 \\
71,6 \\
--.- \\
-\cdots \\
\cdots\end{array}$ & $\begin{array}{l}-\cdots \\
-\cdots \\
75,0 \\
--.\end{array}$ & \begin{tabular}{l}
63,4 \\
-- \\
\hdashline 65,9 \\
71,6
\end{tabular} & $\begin{array}{l}\cdots \\
\cdots \\
\cdots \\
\cdots \\
\cdots\end{array}$ & $\begin{array}{l}\cdots \\
\cdots \\
79,5 \\
\cdots\end{array}$ & $\begin{array}{l}\cdots \\
75,0 \\
\cdots\end{array}$ & \begin{tabular}{l}
56,8 \\
\hdashline 75 \\
75,0
\end{tabular} & $\begin{array}{l}\cdots \\
\cdots \\
\cdots 1,6\end{array}$ & $\begin{array}{l}65,9 \\
\cdots-- \\
\cdots \\
\cdots \\
-\end{array}$ & 75,0 & $\begin{array}{l}71, \overline{6} \\
71,6 \\
79,5 \\
\ldots . .\end{array}$ & $\begin{array}{l}65,9 \\
\cdots \\
-.- \\
-\cdots\end{array}$ & $\begin{array}{l}261,5 \\
265,9 \\
280,5 \\
299,9 \\
297,7\end{array}$ \\
\hline $\begin{array}{r}6 \\
7 \\
8 \\
8 \ldots \ldots \\
9 \\
10 \ldots \ldots\end{array}$ & $\begin{array}{l}\varepsilon 6,8 \\
-48,8 \\
-. . \\
\cdots\end{array}$ & $\begin{array}{l}75,0 \\
\cdots \\
\cdots\end{array}$ & $\begin{array}{l}65,9 \\
65,9 \\
-\cdots \\
71,6\end{array}$ & $\begin{array}{l}\cdots \\
\cdots \\
\cdots \\
\cdots \\
\cdots\end{array}$ & $\begin{array}{l}65,9 \\
68,6 \\
\cdots \\
\cdots\end{array}$ & $\begin{array}{l}\ldots . \\
\cdots 3,4 \\
63,4 \\
\ldots .\end{array}$ & \begin{tabular}{l}
$\ldots .$. \\
\hdashline$\ldots$ \\
$\cdots 3,4$
\end{tabular} & $\begin{array}{l}\cdots- \\
61,1 \\
61,1\end{array}$ & \begin{tabular}{c}
71,6 \\
$\ldots$ \\
\hdashline.- \\
.- \\
-
\end{tabular} & $\begin{array}{l}\cdots \\
\cdots \\
90,0 \\
58,9\end{array}$ & $\begin{array}{l}90,0 \\
90,0 \\
\cdots\end{array}$ & $\begin{array}{l}\cdots .- \\
\cdots \cdots \\
\cdots \\
\cdots \\
\cdots\end{array}$ & \begin{tabular}{c}
65,9 \\
\hdashline.- \\
71,6 \\
.--
\end{tabular} & $\begin{array}{l}260,2 \\
296,8 \\
241,9 \\
315,0 \\
255,0\end{array}$ \\
\hline $\begin{array}{l}11 \ldots \ldots \\
12 \ldots \ldots \\
13 \ldots \ldots\end{array}$ & $\begin{array}{l}\cdots- \\
\cdots- \\
\cdots-\end{array}$ & 71,6 & 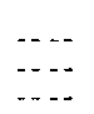 & 63,4 & $\begin{array}{l}75,0 \\
61,1 \\
-\cdots\end{array}$ & 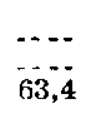 & $\begin{array}{l}30,8 \\
61,1\end{array}$ & $\ldots$ & $\begin{array}{l}68,6 \\
75,0\end{array}$ & $\begin{array}{l}75,0 \\
\cdots\end{array}$ & $\begin{array}{l}\ldots . \\
\cdots \\
\cdots\end{array}$ & $\begin{array}{l}75,0 \\
\cdots \\
\cdots\end{array}$ & 79,5 & $\begin{array}{l}293,6 \\
254,8 \\
271,1\end{array}$ \\
\hline Total .. & 217,2 & 297,7 & 278,4 & 264,3 & 270,6 & 269,7 & 250,3 & 254,0 & 286,8 & 289,8 & 334,5 & 297,7 & 282,9 & 3593,9 \\
\hline
\end{tabular}

A variância teórica de $\varphi$, quando medida em graus, tem o valor de

$$
\mathrm{V}_{\phi}=\frac{820,7}{\mathrm{n}}
$$

A variância residual, obtida a partir dos resultados em aprêço, tem o valor de 46,95 , com 27 graus de liberdade.

Com êsses dados torna-se possível realizar um teste de $\chi^{2}$,

$$
\chi^{2}=\frac{(27)(46,95)(30)}{820,7}=46,33^{*},
$$

cuja probabilidade é de $0,01<\mathrm{P}<0,02$, de forma que, na análise da variância dos resultados transformados, a variância a ser utilizada será a calculada a partir dos mesmos.

Yates analisou experiências dêste tipo de duas maneiras: com e sem recuperação da informação entre-blocos.

\subsection{1 - ANÁLISE SEM RECUPERAÇÃO DA INFORMAÇ̃O ENTRE-BLOCOS}

Usando o primeiro tipo de análise, obtemos, a partir do quadro 2, duas séries de resultados. Os primeiros, que designaremos como $T_{s}$, são os totais correspondentes ao tratamento $\mathbf{s}$. A outra série, designada como $\mathbf{B}_{\mathbf{8}}$, é 
constituída pela so ma dos totais dos blocos que contém o tratamento s. A partir dêsses dois grupos de resultados obtemos os valores $Q_{s}$, onde... $\mathrm{Q}_{\mathrm{s}}=4 \mathrm{~T}_{\mathrm{s}}-\mathrm{B}_{\mathrm{s}}$.

Estes resultados são apresentados no quadro 3 .

Quadro 3.-Cálculo dos valores $B_{s}$, $Q_{s}$ e produções corrigidas $T_{s}^{\prime}$ (sem recuperação da informação entre-blocos), baseado nos ângulos correspondentes ao número de plantas sadias

\begin{tabular}{|c|c|c|c|c|}
\hline Trat:amentos & $\mathrm{T}_{8}$ & $B_{A}$ & Q. & $T^{\prime}$ \\
\hline $\begin{array}{l}0 \\
1 \\
2 \\
3 \\
4\end{array}$ & $\begin{array}{l}217,2 \\
297,7 \\
278,4 \\
264,3 \\
270,6\end{array}$ & $\begin{array}{l}1044,1 \\
1095,3 \\
1111,9 \\
1113,9 \\
1087,1\end{array}$ & $\begin{array}{r}175,3 \\
95,5 \\
-\quad 1,7 \\
-\quad 56,7 \\
-\quad 4,7\end{array}$ & $\begin{array}{l}222,5 \\
305,9 \\
277,0 \\
259,0 \\
275,0\end{array}$ \\
\hline $\begin{array}{l}5 \\
5 \\
6 \\
7 \\
8 \\
8 \\
9\end{array}$ & $\begin{array}{l}269,7 \\
250.3 \\
254,0 \\
286,8 \\
289.8\end{array}$ & $\begin{array}{l}1127,9 \\
1061,4 \\
1060,5 \\
1122,6 \\
1125,1\end{array}$ & $\begin{array}{l}-\quad 49,1 \\
-\quad 60,2 \\
-\quad 44,5 \\
\\
24,6 \\
34,1\end{array}$ & $\begin{array}{l}261,3 \\
257,9 \\
262,8 \\
284,0 \\
286,9\end{array}$ \\
\hline 10 & $\begin{array}{l}334,5 \\
297,7 \\
282,9\end{array}$ & $\begin{array}{l}1190,0 \\
1139,9 \\
1095,9\end{array}$ & $\begin{array}{r}148,0 \\
50,9 \\
35,7\end{array}$ & $\begin{array}{l}322,0 \\
292,1 \\
287,4\end{array}$ \\
\hline Total & 3593,9 & 14.37 .5 .6 & 0,0 & 3593,8 \\
\hline
\end{tabular}

Da maneira usual, obtemos a soma dos quadrados correspondentes a blocos, e a soma dos tratamentos é dada por

$$
\frac{t-1}{\operatorname{rtk}(\mathrm{k}-1)} \mathrm{SQ}_{\mathrm{s}}^{2}=1 / 52 \mathrm{SQ}_{\mathrm{s}}^{2}
$$

A soma correspondente ao resíduo é obtida por diferença. Esstes resultados estão contidos no quadro 4 .

QuADRo 4.-Análise da variância (sem recuperação da informação entre-blocos), baseada nos ângulos correspondentes ao número de plantas sadias

\begin{tabular}{|c|c|c|c|c|}
\hline Origem do êrro & $\begin{array}{l}\text { Graus de } \\
\text { liberdaude }\end{array}$ & $\begin{array}{l}\text { Soma de } \\
\text { quadrados }\end{array}$ & $\begin{array}{l}\text { Quadrado } \\
\text { médio }\end{array}$ & $\mathbf{F}$ \\
\hline $\begin{array}{l}\text { Blocos (nqo ajustados) } \\
\text { Tratamentos (ajustados) } \ldots \ldots \\
\text { Residuo. }\end{array}$ & $\begin{array}{l}12 \\
12 \\
27\end{array}$ & $\begin{array}{l}1490,56 \\
1512,36 \\
1267,56\end{array}$ & $\begin{array}{r}126,03 \\
46,95\end{array}$ & … \\
\hline rotal $\ldots \ldots$ & .51 & 4270,48 & $\ldots \ldots$ & \\
\hline
\end{tabular}

Os totais ajustados, correspondentes aos tratamentos, são obtidos a partir da fórmula a seguir, onde $\mathrm{G}$ corresponde ao total gerál encontrado para o experimento. 


$$
\mathrm{T}^{\prime \prime}-\left(1 / \mathrm{t}+\frac{(\mathrm{t}-1) \mathrm{Q}_{\mathrm{s}}}{\mathrm{t}(\mathrm{k}-\mathrm{-}-1)}=276,5+4 / 13 \mathrm{Q}_{\mathrm{s}} .\right.
$$

A variâneia correspondente à produão toblal, ajustada, relativa a um tratamento, e de $16,95 \times 12 / 13=57,78$.

Convém notar que êste tipo de análise da variância conduz a um teste cxato das médiats de tratamento.

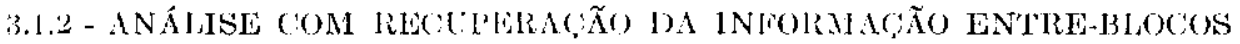

Consideremos, agora, a análise da variânelia com recuperação da infor-

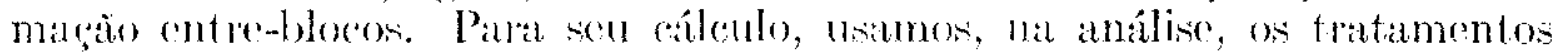
nãu ajustalos, cuja somat de quadrados é obtida da maneira usual. A somat

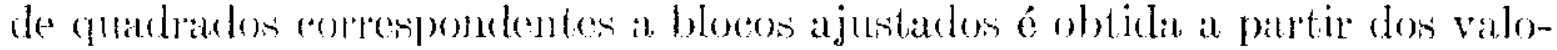

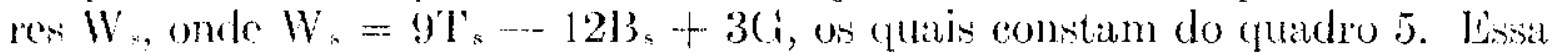
soma de cuadrados é:

$$
\frac{s W_{s}^{2}}{t+(t--k)(k-1)}=\frac{s W^{2}}{1101}==(120, \pi, \pi .
$$

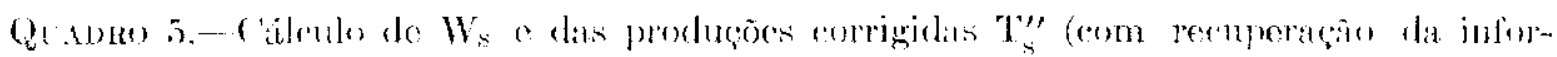

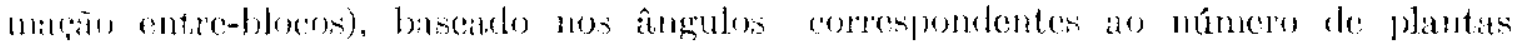
sildiats

\begin{tabular}{|c|c|c|c|c|}
\hline$W_{s}$ & $T^{\prime \prime}{ }_{s}$ & Trat:tumento & & $\mathrm{T}{ }^{\prime \prime}$ \\
\hline $\begin{array}{r}207,3 \\
317,4 \\
5 \%, 5 \\
207+4 \\
171,9 \\
325,8 \\
297,6\end{array}$ & $\begin{array}{l}217,7 \\
298,5 \\
278,3 \\
263,8 \\
271,0 \\
238,0 \\
25,1,0\end{array}$ & 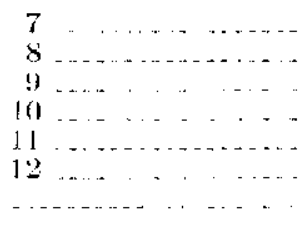 & 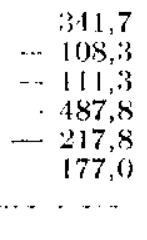 & 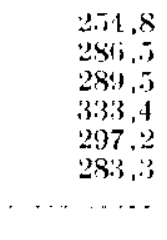 \\
\hline
\end{tabular}

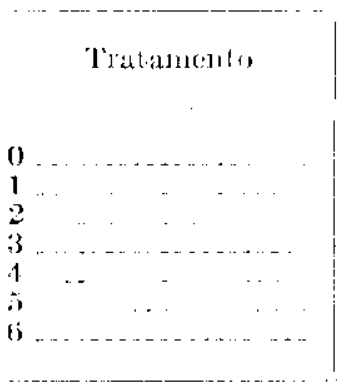

(); resultudos obtides para a anólise da varjância são os do cuatdro 6 .

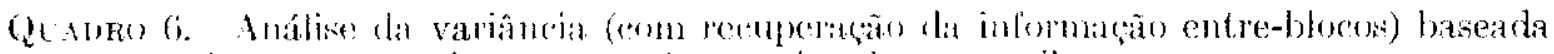

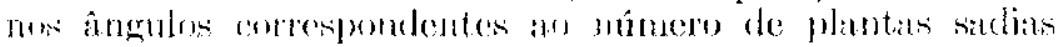

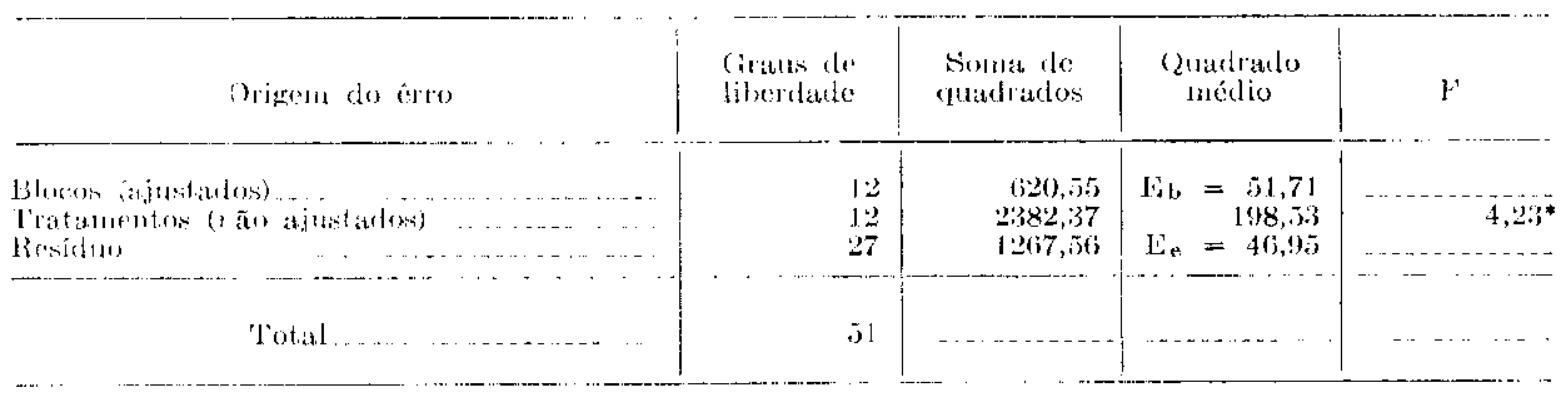

Paral ohter os tratamentos ajustados, nasmos o fator de ponderasäo

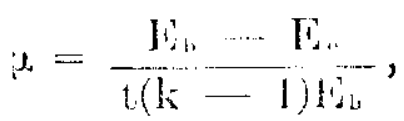


onde $\mathrm{E}_{\mathrm{b}}$ e $\mathrm{E}_{\mathrm{e}}$ são os quadrados médios indicados no quadro $6 \mathrm{e}$, por conseguinte,

$$
\mu=\frac{4,76}{39(51,71)}=0,0024 \text { e } \quad \mathrm{T}_{\mathrm{s}}^{\prime \prime}=\mathrm{T}_{\mathrm{s}}+\mu \mathrm{W}_{\mathrm{s}} .
$$

A variância para o teste dos tratamentos $\mathbf{T}^{\prime \prime}$ é dada por $\mathbf{E}_{\mathrm{e}}[1+(\mathrm{t}-$ - k) $\mu]=47,97$.

O teste de $\mathbf{F}$ relativo a tratamentos ajustados é, neste caso, uma prova aproximada.

\section{2 - ANÅLISE DA PRODUÇÃ DE TOMATES}

Consideramos, agora, os resultados de produção de tomates, incluídos no quadro 7 .

QUADRo 7.-Disposição dos tratamentos no campo e produção de tomates por parcela

\begin{tabular}{|c|c|c|c|c|c|c|c|c|c|}
\hline \multirow{2}{*}{ Bloco } & \multicolumn{8}{|c|}{ Tratamentos e produções respectivas } & \multirow{2}{*}{$\begin{array}{l}\text { Produ- } \\
\text { ção total }\end{array}$} \\
\hline & Trat. & Prod. & Trat. & Prod. & 'I'rat. & Prod. & Trat. & Prod. & \\
\hline & & $k g$ & & $\mathrm{~kg}$ & & $k g$ & & kg & \\
\hline 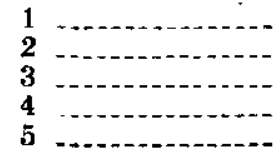 & $\begin{array}{r}0 \\
11 \\
11 \\
3 \\
10\end{array}$ & $\begin{array}{l}\mathbf{6 2 , 5} \\
\mathbf{7 7 , 6} \\
\mathbf{6 2 , 9} \\
\mathbf{5 4 , 6} \\
\mathbf{5 6 , 5}\end{array}$ & $\begin{array}{l}1 \\
1 \\
6 \\
2 \\
8\end{array}$ & $\begin{array}{l}64,8 \\
65,1 \\
53,0 \\
56,9 \\
50,7\end{array}$ & $\begin{array}{r}3 \\
7 \\
10 \\
5 \\
3\end{array}$ & $\begin{array}{l}58,6 \\
34,4 \\
45,0 \\
53,6 \\
51,8\end{array}$ & $\begin{array}{r}9 \\
12 \\
0 \\
11 \\
7\end{array}$ & $\begin{array}{l}41,2 \\
59,1 \\
53,6 \\
53,4 \\
39,6\end{array}$ & $\begin{array}{l}227,1 \\
236,2 \\
214,5 \\
218,5 \\
198,6\end{array}$ \\
\hline $\begin{array}{r}\mathbf{6} \\
\mathbf{7} \\
\mathbf{8}\end{array}$ & $\begin{array}{r}2 \\
10 \\
5 \\
5 \\
2\end{array}$ & $\begin{array}{l}51,9 \\
42,9 \\
30,7 \\
46,3 \\
51,3\end{array}$ & $\begin{array}{r}\mathbf{0} \\
2 \\
0 \\
10 \\
9\end{array}$ & $\begin{array}{l}36,4 \\
36,6 \\
22,4 \\
38,9 \\
28,7\end{array}$ & $\begin{array}{r}12 \\
1 \\
7 \\
12 \\
7\end{array}$ & $\begin{array}{l}48,7 \\
42,8 \\
32,4 \\
44,2 \\
28,1\end{array}$ & $\begin{array}{l}8 \\
4 \\
4 \\
9 \\
6\end{array}$ & $\begin{array}{l}34,0 \\
31,5 \\
27,1 \\
41,5 \\
28,2\end{array}$ & $\begin{array}{l}171,0 \\
153,8 \\
112,6 \\
170,9 \\
136,3\end{array}$ \\
\hline $\begin{array}{l}11 \\
12 \\
13\end{array}$ & $\begin{array}{l}8 \\
6 \\
6\end{array}$ & $\begin{array}{l}15,7 \\
22,3 \\
47,9\end{array}$ & $\begin{array}{r}9 \\
12 \\
1\end{array}$ & $\begin{array}{l}\mathbf{4 4 , 4} \\
\mathbf{5 5 , 7} \\
\mathbf{5 7 , 5}\end{array}$ & $\begin{array}{r}11 \\
4 \\
5\end{array}$ & $\begin{array}{l}50,4 \\
56,2 \\
51,7\end{array}$ & $\begin{array}{l}4 \\
3 \\
8\end{array}$ & $\begin{array}{l}54,6 \\
51,3 \\
44,5\end{array}$ & $\begin{array}{l}165,1 \\
185,5 \\
201,6\end{array}$ \\
\hline
\end{tabular}

As produções dos tratamentos 6 - bloco 12 e 8 - bloco 11 , foram de $22,3 \mathrm{~kg}$ e $15,7 \mathrm{~kg}$, respectivamente. Tais resultados, especialmente o último, destoam dos obtidos nas outras repetições. A discrepância é explicada pelo aparecimento de mancha onde houve ataque intenso de afídios. $\underset{\mathrm{E}}{\text { razoável }}$ admitir que, no presente caso, a baixa produção obtida nos canteiros considerados tem uma causa determinada e assim podemos considerar independente do efeito dos inseticidas empregados, razão pela qual resolvemos substituir êsses resultados por produções calculadas.

A solução que torna mínimo o êrro entre-bloco é a seguinte (1) :

$$
x=\frac{\operatorname{tr}(k-1) B_{s}+k(t-1) Q_{.}-(t-1) Q^{\prime}}{(k-1)[(k-1)-k(t-1)]},
$$

onde $\mathrm{Q}^{\prime}$. é a soma dos valores $\mathrm{Q}_{\mathrm{i}} ; \mathbf{i}$ indica os valores correspondentes a tratamentos outros que s e constantes do bloco que contém a produção procurada. 
No presente caso necessitamos calcular dois valores : $\mathbf{x}$ que é a produção do tratamento 8 , bloco 11 , e y produção do tratamento 6 , bloco 12 .

Quadro 8.-Valores de $T_{\mathrm{A}}$ e $B_{\mathrm{S}}$ e produções corrigidas $T_{\mathrm{S}}^{\mathfrak{r}}$ (sem recuperação da informação entre-blocos). Produções totais em quilogramas

\begin{tabular}{|c|c|c|c|c|}
\hline Tratamento & $T_{B}$ & $\mathrm{~B}_{\mathrm{B}}$ & $T^{\prime}$ & $\mathrm{T}^{\prime \prime}$ \\
\hline $\begin{array}{l}0 \\
0 \\
1\end{array}$ & $\begin{array}{l}174,9 \\
230,2 \\
196,7 \\
216,3 \\
169,4\end{array}$ & $\begin{array}{l}725,2 \\
818,7 \\
679,6 \\
807,4+y \\
579,0+x+y\end{array}$ & $\begin{array}{l}179,1 \\
218,4 \\
220,0 \\
192,1 \\
193,6\end{array}$ & $\begin{array}{l}178,2 \\
220,9 \\
215,1 \\
197,1 \\
188,5\end{array}$ \\
\hline $\begin{array}{l}5 \\
5 \\
6\end{array}$ & $\begin{array}{l}182,3 \\
129,1+y \\
134,5+5 \\
129,2+x \\
155,8\end{array}$ & $\begin{array}{l}703,6 \\
715,6+y \\
683,7 \\
720,6+x \\
683,7+x\end{array}$ & $\begin{array}{l}194,9 \\
163,8 \\
142,2 \\
157,4 \\
157,3\end{array}$ & $\begin{array}{l}192,3 \\
165,2 \\
140,6 \\
159,0 \\
157,0\end{array}$ \\
\hline 10 & $\begin{array}{l}183,3 \\
244,3 \\
207,7\end{array}$ & $\begin{array}{l}737,8 \\
818,6+x \\
741,3+y\end{array}$ & $\begin{array}{l}185,6 \\
224,7 \\
201,8\end{array}$ & $\begin{array}{l}185,1 \\
228,8 \\
203,1\end{array}$ \\
\hline
\end{tabular}

A partir dos resultados do quadro 8 , atribuindo a $\mathbf{y}$ um valor provisório $129,1 / 3=43,0$, calculamos :

$$
\mathrm{x}=\frac{13 \mathrm{~B}_{\mathrm{s}}+4 \mathrm{Q}_{\mathrm{a}}-\mathrm{Q}_{\mathrm{s}}^{\prime}}{27}=
$$

$=\frac{13(149,4)+4[4(129,2)-720,6]}{27}$

$=\frac{4(169,4+155,8+244,3)-(622+683,7+818,6)}{27}=36,0$.

Usando êste valor de $\mathbf{x}$, obtemos $\mathbf{y}$, por processo idêntico ao indicado : $\mathrm{y}=1114,9 / 27=41,3$.

Utilizando, agora, êste valor de $\mathbf{y}$, recalculamos $\mathbf{x}$. Obtemos o valor $\mathbf{x}^{\prime}=970,0 / 27=35,9$.

Se, agora, dermos a $\mathbf{x}$ êste último valor e recalcularmos $\mathbf{y}$, obteremos $\mathbf{y}^{\prime}=41,3$, que é o mesmo resultado já encontrado. Desta forma podemos terminar aqui êste processo de iteração. Os valores obtidos são agora utilizados no cálculo da análise da variância. Em primeiro lugar, será considerada a análise sem recuperação da informação entre-blocos.

As produções corrigidas, quando não usamos a informação entre-blocos (valores $\mathrm{T}^{\prime}$ ) e quando a utilizamos (valores $\mathrm{T}^{\prime \prime}$ ), foram incluidas no quadro 8. Podemos verificar que na maioria dos casos essas produçóes corrigidas pouco diferem das obtidas no experimento.

$\mathrm{Na}$ análise da variância (quadro 9), devido à utilização de duas produções teóricas, perdemos dois graus de liberdade, correspondentes à soma residual de quadrados. 
Quadro 9.-Análise da variância (sem recuperaçao da informação entre-bloco). I'rodução por parcela em cquilogramats

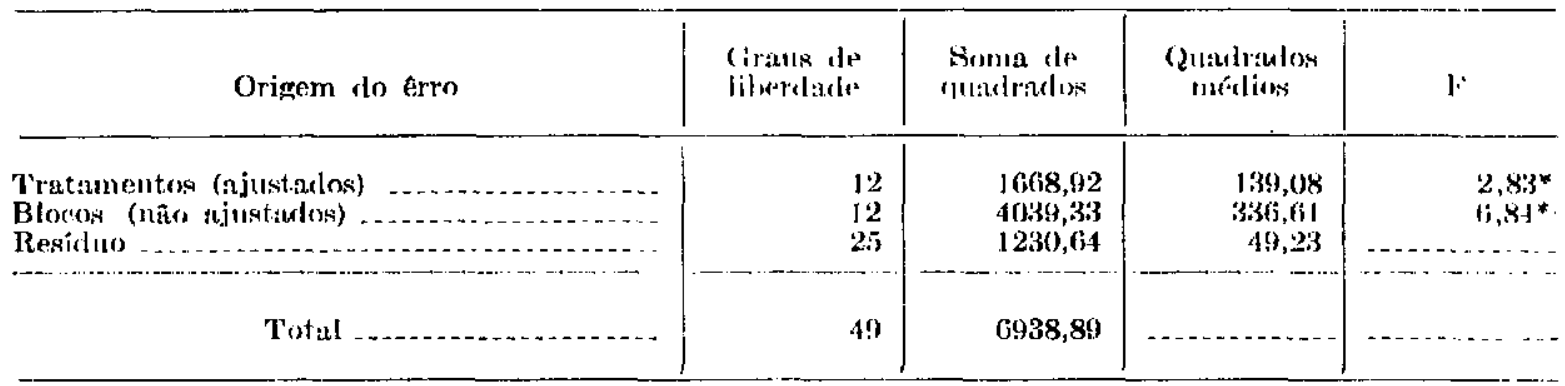

A variância residual efetiva, a ker usada na comparaģão de trattamentós, é $4 \% / 13(49,23)=60,59$.

Com a recuperação da informaceño entre-blocos, obtemos para essa variância o valor de 58,22 (quidro io).

Quadro 10.-Análise da variância (com recuperaciōo da informanaino entréplocos). Produção por parcela em quilogramas

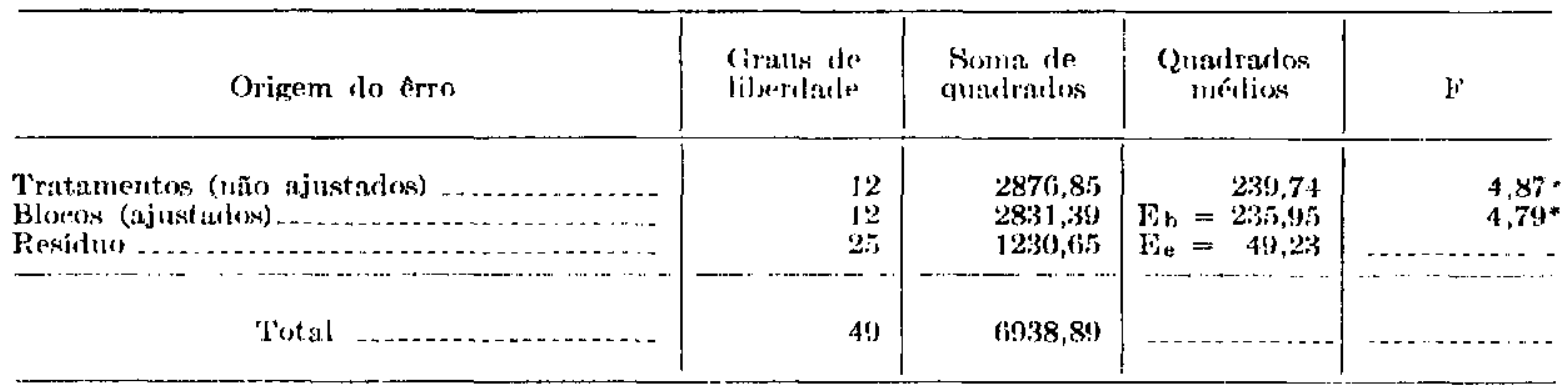

\section{4 - SUMĀRIO E CONCLUSÕES}

A análise da variância com recuperação da informaçĩo entre-blocos é indicada nos ensaios com um número de tratamentos relativamente grande. No caso em aprêço êsse número foi do 13 e obtivemos as seguintes variâncias residuais, em ângulo correspondente ao número de plantas sadias : 57,78 sem recuperação da informação entre-blocos e 47,97 com recuperação da informação entre-blocos. A redução obtida com a recuperação da informação entre-blocos foi de $16,92 \%$. Para a prodıção de tomates, obtivemos 60,59, na análise sem reeuperação da informaç̃o entre-blocos e 58,22 , na com recuperação da informição entre-blocos.

$\Lambda$ redução obtida com a recuperação da informaçĩo entre-blocos foi de $3,91 \%$.

De acôrdo com êsses resultados, sòmente para o caso do número de plantas é que se mostrou realmente vantajoso o emprêgo do segundo processo da análise.

Representamos, na figura 1 , os resultados obtidos nas análises anteriores. Os gráficos se referem a totais de tratamentos depois de ajustados, fazendo-se uso da informação entre-lolocos. O gráfico superior se refere a 


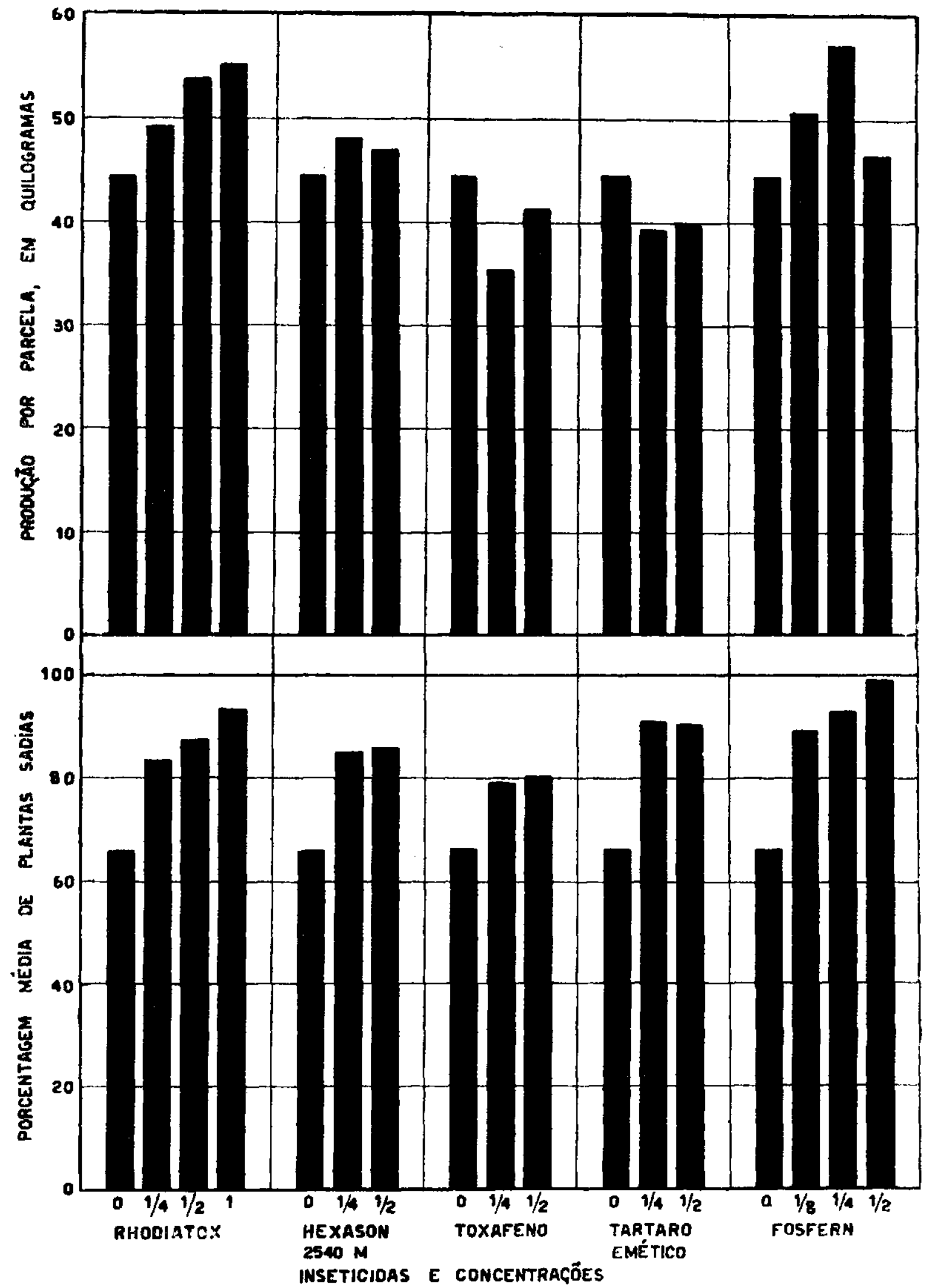

Figrer 1.-Infhuência de vários inseticidas comerciais, em diferentes eoneentraçũes, no eoutrôle do vira-cabega do tomateiro. Resultados relativos à produçăo e à percentagem de plantas sailias. 
ângulo correspondente ao número de plantas sadias, e o inferior à produção de tomates em quilogramas.

Estes últimos resultados permitem concluir que, de maneira geral, o contrôle obtido é proporcional às concentrações dos inseticidas empregados e também que êsses inseticidas, em suas concentraçóes altas, afetam o desenvolvimento das plantas, fatos já notados nas experiências anteriores. Assim, nas condições em que foi realizada esta experiência, isto é, em ocasião em que o ataque de vira-cabeça foi relativamente moderado, os tratamentos podem ser considerados como pertencendo a dois grupos : $1 .^{\circ}$, aquêles que agiram favoràvelmente, isto é, em que ao ganho em plantas sadias correspondeu um aumento de produção em relação à média - Rhodiatox, Hexason e Fosfern ; e 2. ${ }^{\circ}$, aquêles em que, apesar de ter havido um aumento significante no número de plantas sadias, não houve um aumento correspondente da produção - Tártaro emético e Toxafeno.

Sossivel verificar essas conclusões a partir dos resultados apresentados no quadro 11.

Qtadro 11.-Decomposição da soma de quadrados correspondentes aos tratamentos.

(Totais ajustados, sem recuperação da informação entre-blocos.)

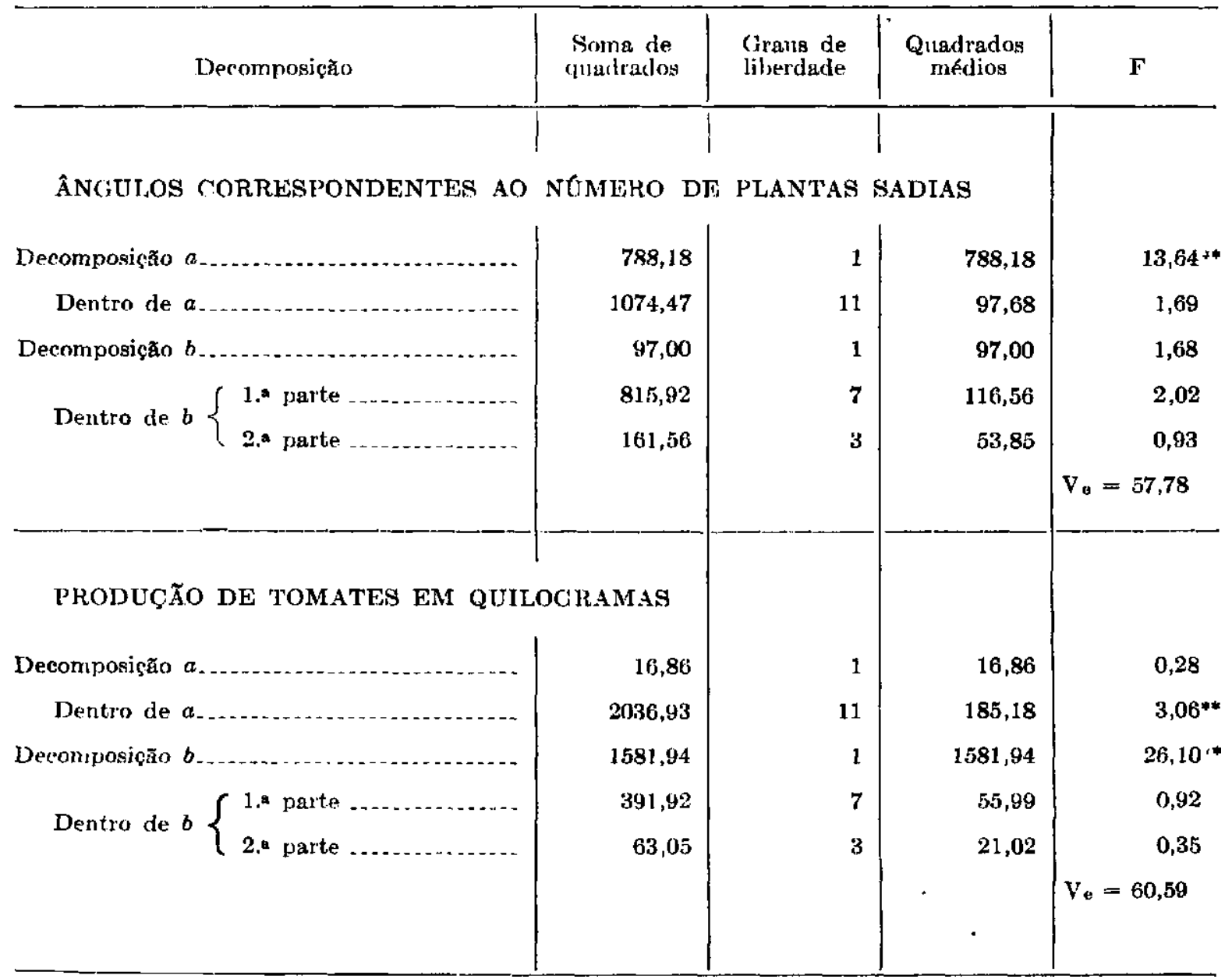


As decomposições usadas foram as seguintes:

Decomposição $a$ : Comparação da testemunha com os tratamentos empregados.

Dentro de $a$ : Comparação dos tratamentos excluindo a testemunha.

Decomposição $b$ : Tratamentos com Rhodiatox, Hexason 2540 M e Fosfern (1." grupo) comparados aos tratamentos com Toxafeno e Tártaro emético (2. grupo).

Dentro de b, 1." parte: Comparação entre os tratamentos do primeiro grupo. Dentro de $b, 2 .^{a}$ parte : Comparação entre os tratamentos do segundo grupo.

Os tratamentos inclúdos no primeiro grupo (Rhodiatox e Fosfern) correspondem a inseticidas preparados com um mesmo princípio ativo, e é desnecessário um teste para verificar que, para as mesmas concentrações dêsse princípio (o tiofosfato de dietil paranitrofenila), êles podem ser considerados como equivalentes. Não é possivel afirmar que, quanto a seu comportamento, dêles difere o Hexason $2540 \mathrm{M}$.

Limitando-nos aos dois primeiros inseticidas, e deixando de lado sua marca comercial, para considerá-los ùnicumente em função do princípio ativo empregado, podemos estudar êsses tratamentos como segue :

Conchntração him 'ilofosfato

DE DIETIL PARANITROFENILA

Testemunha

$1: 80.000$

$1: 40.000$

$1: 20.000$

$1: 10.000$

\section{Tratamento}

$\begin{array}{rrr} & & 0 \\ & 3 \\ 2 & \text { e } & 12 \\ 1 & \text { e } & 11 \\ & & 10\end{array}$

Estes resultados esclarecem que, para maior concentração utilizada, êsse inseticida é prejudicial e que, no caso de o veículo utilizado nos dois preparados não diferir de maneira essencial, o dano causado deve ser atribuído exclusivamente ao princípio ativo.

A regressão linear, entre os pontos fornecidos pelos tratamentos considerados, não elimina pràticumente variação alguma, enquanto quc é altamente significativa a redução devida à regressão cuadrática (quadro 12)

Quadro 12.-Prova de significância de afastamento da regressão linear. Produção total de tomates, por tratamento, em quilogramas (produções ajustadas com recuperação entre-blocos)

\begin{tabular}{|c|c|c|c|c|}
\hline Origem de variaşũo & $\begin{array}{l}\text { Graus d: } \\
\text { libcrdacle }\end{array}$ & $\begin{array}{l}\text { Somes de } \\
\text { quedradus }\end{array}$ & $\begin{array}{l}\text { Quadrados } \\
\text { médios }\end{array}$ & $\mathbf{F}$ \\
\hline $\begin{array}{l}\text { Total } \\
\text { Regressão linear } \\
\text { Resto (1) } \\
\text { Regressắo quadrátín } \\
\text { Resto (2) }\end{array}$ & $\begin{array}{l}6 \\
1 \\
5 \\
1 \\
4\end{array}$ & $\begin{array}{r}2095,12 \\
18,93 \\
2076,17 \\
3928,06 \\
148,11\end{array}$ & $\begin{array}{r}1928,09 \\
37,03\end{array}$ & 52,07 \\
\hline
\end{tabular}




\section{SUMMAIRY}

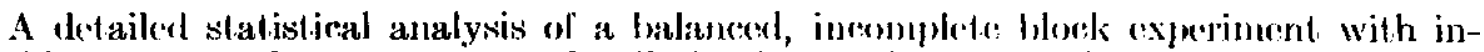
serticides to control tomato spotted wilt is given. The effent of the treatments was studied in relation to stand an yicld. The angular transfomation was used for stand, and the analysis of stand and yield was made with and without the inter-block information.

The results indicated that all insecticides tested, viz. Rhorliatox, Fesfern, Ifexasm $25.10 \mathrm{M}$, Toxaphene, and tartar emetic reduesel the number of plants killed by spotted wilt. The higher the coneentration of the insedteides the better was the gain in stand. Some insecticides had a detrimental effect on the plant al high concentrations.

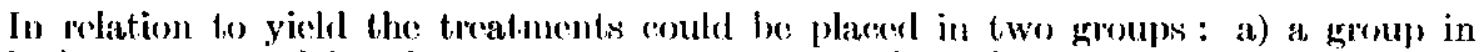
which demage caused by the treatment was grealer than the benefit: resulting from a better stand with 110 gain in yicld; b) a group in which there was also a gain in yield.

\section{IITEIRA'TU!RA CIITADA}

1. Cochran, W. G. c Cox, G. M. Ém T'he experinental designs. pág. 322-324. 1." edição, ,Iohn Wiley \& Sons, Nova Ior(que, 1950.

2. Costa, A. S., Forster, R. e Fraga Jr., C. C: Contrôle de vira-cabeça do tomate pula destruiçấo do vetor. Birugantia 10 : 1-9, fig. 1. 1950.

3. Fisher, R. A. \& Yates, F. Em Statistical lables lor hiological, agricultural and medieal rescarch pág. 12, 1." edição. ()liver and Boyd, Londres, 1938.

4. Mather, K. Hm Statistical analysis in ljology, jág. 23-1-23y, 2." ediçä́, Interseience Publishers Inc. Nova Iorque, 1917. 